\title{
Stellar yields with rotation and their effect on chemical evolution models ${ }^{\star}$
}

\author{
C. Chiappini ${ }^{1}$, F. Matteucci ${ }^{2}$, and G. Meynet ${ }^{3}$ \\ 1 Osservatorio Astronomico di Trieste, via G. B. Tiepolo 11, 34131 Trieste, Italia \\ 2 Dipartimento di Astronomia, Universita' degli Studi di Trieste, via G. B. Tiepolo 11, 34131 Trieste, Italia \\ 3 Geneva Observatory, 1290 Sauverny, Switzerland
}

Received 26 May 2003 / Accepted 29 July 2003

\begin{abstract}
We compute the evolution of different abundance ratios in the Milky Way (MW) for two different sets of stellar yields. In one of them stellar rotation is taken into account and we investigate its effects on the chemical evolution model predictions. Moreover, we show that some abundance ratios offer an important tool to investigate the halo-disk discontinuity. For the first time it is shown that the effect of a halt in the star formation between the halo/thick disk and thin disk phases, already suggested from studies based both on $\mathrm{Fe} / \mathrm{O}$ vs. $\mathrm{O} / \mathrm{H}$ and $\mathrm{Fe} / \mathrm{Mg}$ vs. $\mathrm{Mg} / \mathrm{H}$, should also be seen in a $\mathrm{C} / \mathrm{O}$ versus $\mathrm{O} / \mathrm{H}$ plot if $C$ is produced mainly by low-and intermediate-mass stars (LIMS). The idea that C originates mainly from LIMS is suggested by the flat behavior of the $[\mathrm{C} / \mathrm{Fe}]$ ratio as a function of metallicity, from $[\mathrm{Fe} / \mathrm{H}] \sim-2.2$ to solar, and by the fact that very recent $\mathrm{C} / \mathrm{O}$ measurements for stars in the MW halo and disk seem to show a discontinuity around $\log (\mathrm{O} / \mathrm{H})+12 \sim 8.4$. Finally, a more gentle increase of $\mathrm{N}$ abundance with metallicity (or time), relative to models adopting the yields of van den Hoek \& Groenewegen (1997), is predicted by using the stellar yields of Meynet \& Maeder (2002 - which include stellar rotation but not hot-bottom burning) for intermediate mass stars. This fact has some implications for the timescales of $\mathrm{N}$ enrichment and thus for the interpretation of the nature of Damped Lyman Alpha Systems.
\end{abstract}

Key words. Galaxy: abundances - Galaxy: evolution - Galaxy: formation

\section{Introduction}

In the past years new observations have suggested that the Milky Way (MW) formed in a more complex way than the pure dissipational collapse suggested originally by Eggen et al. (1962, ELS). Their model assumed a continuous evolutionary transition in the formation of the halo, thick disk and the thin disk. Apart from dynamical arguments (Wyse \& Gilmore 1992), the chemical properties of the MW disk are convincingly showing us that its formation was neither smooth nor continuous. As an example, Gratton et al. $(1996,2000)$ presented a compilation of stars for which both the kinematics and the $[\mathrm{Fe} / \mathrm{O}]$ abundance ratios were known. They observed for the first time a discontinuity in the $[\mathrm{Fe} / \mathrm{O}]$ vs. $[\mathrm{O} / \mathrm{H}]$ plot which was interpreted as a halt in the star formation before the thin disk formation. In particular, they observed that around $[\mathrm{O} / \mathrm{H}]=-0.2$ dex (in solar scale) the oxygen abundance remains constant whereas the $[\mathrm{Fe} / \mathrm{O}]$ ratio keeps increasing. This is interpreted as a break in the star formation since in this case oxygen would not be produced whereas Fe would continue to originate from long-living systems giving rise to type Ia SNe. In this scenario no stars would have been formed during the gap and this implies that a gap should also be seen in an $[\mathrm{Fe} / \alpha]$ vs. $[\alpha / \mathrm{H}]$ diagram. The same behavior was found by

\footnotetext{
* The figures are available in color in electronic form.
}

Fuhrmann (1998) for $[\mathrm{Fe} / \mathrm{Mg}]$ vs. $[\mathrm{Mg} / \mathrm{H}]$. However, this should still be confirmed by larger data samples.

Chiappini et al. (1997) have shown that a two-infall model for the formation of the MW, where the halo (and part of the thick disk) formed on a short timescale whereas the thin-disk formed on a much longer one, can explain not only the above observations but also provides a good fit to the G-dwarf metallicity distribution in the solar vicinity (see also Kotoneva et al. 2002). In the present work we argue that, if we believe that there was such a halt in the star formation rate (SFR) and if the carbon enrichment of the interstellar medium (ISM) is mainly due to low and intermediate mass stars (LIMS) and hence is produced on long timescales as iron, the same kind of discontinuity observed for $\mathrm{Fe} / \mathrm{O}$ and $\mathrm{Fe} / \mathrm{Mg}$ should be seen in a $\log (\mathrm{C} / \mathrm{O})$ vs. $\log (\mathrm{O} / \mathrm{H})$ diagram (Chiappini et al. 2003 CRM2003).

However, the origin of $\mathrm{C}$ (and $\mathrm{N}$ ) we see today in the ISM is still an open problem. In particular, while Henry et al. (2000) favor the hypothesis that most of the carbon we observe today in the ISM comes from massive stars, CRM2003 suggest that most of the carbon comes from LIMS. These conclusions are very dependent on the adopted stellar yields. Many are the processes involved in the computation of the stellar yields of $\mathrm{C}$ and $\mathrm{N}$ and there are still many uncertainties present in these calculations (see Meynet \& Maeder 2002 - MM). MM have 
shown that stellar rotation can affect the predictions of the stellar yields especially for $\mathrm{He}, \mathrm{C}, \mathrm{N}$ and $\mathrm{O}$. Chemical evolution models can thus be used to test and constrain the stellar yields, as it will be shown in the next sections. In particular, we will evaluate the impact of the new stellar yields of MM on important open questions related to the $\mathrm{C}, \mathrm{N}$ and $\mathrm{He}$ enrichment in galaxies. Moreover, we discuss how the abundance ratios depend on the adopted stellar yields and also how they can be used to infer the star formation history of our Galaxy and the nature of Damped Lyman Alpha systems (DLAs).

\section{Stellar yields}

In this work we compare the following two different models: a) a model computed with van den Hoek \& Groenewegen (1997 $\mathrm{vdHG})$ yields for LIMS and Woosley \& Weaver (1995 - WW) yields for massive stars (model A) and b) a model computed with the recent published stellar yields of Meynet \& Maeder (2002 - MM), which take into account the effects of rotation on stellar evolution, for the whole range of stellar masses (model B). In model A the carbon yields are multiplied by a factor of three in the 40-100 $M_{\odot}$ mass range ${ }^{1}$.

Three important facts should be noted with respect to the different sets of yields:

- The vdHG yields we adopt here are for the case of $\eta_{\mathrm{AGB}}$ (the mass loss parameter during AGB phase) varying with metallicity $\left(\eta_{\mathrm{AGB}}=1\right.$ and 2 for $Z=0.001$ and 0.004 respectively, and $\eta_{\mathrm{AGB}}=4$ for $Z=0.008,0.02$ and 0.04 ). We notice that a lower value for $\eta_{\mathrm{AGB}}$ implies a larger yield of carbon. This is because a lower mass loss leads to a longer lifetime and hence more thermal pulses. As a consequence, more $\mathrm{C}$ is dredged up to the stellar surface. An example of the impact of the mass loss parameter (which is one of the main uncertainties in the computation of $\mathrm{vdHG}$ yields, together with the efficiency of the hot bottom burning - HBB) is shown in Fig. 6 (upper left panel). The dashed line shows a model computed with vdHG yields for $Z=0.001$ and $\eta_{\mathrm{AGB}}=1$ for all metallicities. In this case a high $\log (\mathrm{C} / \mathrm{O})$ ratio is predicted at the present time (see a detailed discussion in the Sect. 4).

\footnotetext{
${ }^{1} \mathrm{WW}$ computed the stellar yields for stars in the 11 to $40 M_{\odot}$ mass range. For masses larger than $40 M_{\odot}$ we extrapolated their stellar yields, as shown by the triangles in Figs. 1 and 3. In particular, for carbon, our extrapolated value at $70 M_{\odot}$ was chosen to match the value computed for this mass by Nomoto et al. (1997 - these latter are essentially the same as in Thielemann et al. 1996, but for an enlarged grid of masses - see open triangle and open circle in Fig. 1 for a $70 M_{\odot}$ ). However, as concluded in CMR2003, although the yields of Thielemann et al. (1996) ensure a good agreement between data and model predictions for many abundance ratios, for carbon it was necessary to increase the stellar yields, for $m>40 M_{\odot}$, by a factor of three. In fact, Thielemann et al. (1996) do not account for mass loss which, during the WC phase, is responsible for ejecting helium and carbon into the interstellar medium. Given this fact, in the present work we also increased the WW yields (which also do not account for mass loss) by the same factor (see filled triangles in the upper panel of Fig. 1).
}

- MM use a different approach from vdHG. While the latter authors computed their yields by means of synthetic AGB models, MM yields were obtained from self-consistent complete stellar models (without any fine tuning of parameters related to the so called HBB). MM were able to show that rotation opens a new alternative for primary $\mathrm{N}$ production in intermediate mass stars, in addition to the classical HBB scenario. Moreover they also predict some primary $\mathrm{N}$ production in massive stars. However, for the intermediate mass star models MM calculations stop at the beginning of the thermal-pulse AGB phase (TP-AGB). The third dredge-up (and the HBB) would occur in more evolved stages and thus are not included in the MM results. Only, in the case of models with $Z=10^{-5}$ they do obtain the third dredge-up in the sense that the stellar surface becomes enriched both in $\mathrm{H}$ and He-burning products. Forthcoming results (Meynet, private communication) will include the more evolved phases of stellar evolution thus predicting not only the 3rd dredge up contribution to ${ }^{12} \mathrm{C}$ but also obtaining the HBB effect as a natural consequence of the stellar evolution itself, without any parametrization. In fact, HBB may still appear and thus add its contribution to the synthesis of primary $\mathrm{N}$.

- WW models included the explosive nucleosynthesis, but did not include rotation/mass loss. Their tabulated yields, differently from the ones given in MM (or Thielemann et al. 1996 - TNH) include not only the processed material (i.e., the new elements produced and released by a given star of mass $m$ ), but also the unprocessed material of the stellar envelope. This last quantity has to be subtracted from their tables if one wants to use their stellar yields in a consistent way.

In summary, one has to keep in mind that the stellar yields of $\mathrm{MM}$ offer a new alternative for the primary $\mathrm{N}$ production in intermediate mass stars and in massive stars. However, their results do not include the third dredge-up and hot-bottom burning and thus their yields of $\mathrm{C}$ and $\mathrm{N}$ for the intermediate mass range should be taken as lower limits. On the other hand, the vdHG yields, which include both HBB and 3rd dredge-up, depend strongly on the $\eta_{\mathrm{AGB}}$ and HBB efficiency parameters adopted in their synthetic models and are thus very uncertain.

Although MM did not formally include the third dredgeup, we think it is worth studying the effects of their new yields on chemical evolution models for the following reasons: a) MM yields for nitrogen at low metallicity results from a new process whose importance for chemical evolution has to be studied. In absence of a real quantitative assessment of the importance of the HBB it appears to us interesting to study the importance of this new process, which produce "non-parametric" yields, independently of HBB and b) this is particularly justified in view of the fact that this new process give primary $\mathrm{N}$ yields at low metallicity not very different from those obtained from parametric studies as the one of van den Hoek and Groenewegen. This questions the importance of the HBB. Only by studying the effects separately it will be possible to understand the different consequences of the two processes. 
Table 1. Meynet \& Maeder yields for $Z=0.020$ and $V_{\text {rot }}=$ $300 \mathrm{~km} \mathrm{~s}^{-1}$.

\begin{tabular}{lllll}
\hline \hline Initial mass & $m p^{4} \mathrm{He}$ & $m p^{{ }^{12} \mathrm{C}}$ & $m p_{14 \mathrm{~N}}$ & $m p^{{ }^{12} \mathrm{O}}$ \\
\hline 120 & 31.347 & 7.114 & 0.80 & 7.00 \\
60 & 15.269 & 2.161 & 0.35 & 3.48 \\
40 & 5.764 & 1.732 & 0.13 & 3.80 \\
25 & 2.525 & 0.310 & 0.12 & 2.88 \\
20 & 2.621 & 0.178 & 0.11 & 1.41 \\
15 & 2.201 & 0.099 & 0.09 & 0.41 \\
12 & 1.761 & 0.014 & 0.07 & 0.07 \\
9 & 1.056 & 0.055 & 0.03 & 0.017 \\
7 & 0.744 & 0.037 & 0.02 & 0.011 \\
5 & 0.433 & 0.018 & 0.014 & 0.005 \\
3 & 0.121 & .00031 & .0065 & -0.0014 \\
2 & 0.067 & .0001 & .0025 & -0.0014 \\
\hline
\end{tabular}

\subsection{The yields adopted in this work}

We are interested to study the effects of stellar yields which account for stellar rotation on chemical evolution models. In model B we adopted the yields of MM for stellar rotational velocities of $300 \mathrm{~km} \mathrm{~s}^{-1}$ (MM published only the tables for $Z=10^{-5}$ and 0.004 . Their computed values for $Z=0.020$ and $V_{\text {rot }}=300 \mathrm{~km} \mathrm{~s}^{-1}$ are shown in Table 1 . These yields have been deduced from the models of Meynet \& Maeder 2000). MM provide also calculations for larger velocities $\left(400 \mathrm{~km} \mathrm{~s}^{-1}\right)$ but only for some specific masses and metallicities, namely: for $\mathrm{N}$ at $Z=0.004$ (for a mass of $20 M_{\odot}$ ) and for $\mathrm{C}, \mathrm{N}$ and $\mathrm{O}$ for $Z=10^{-5}$ (for stars of 9 and $20 M_{\odot}$ ). In the case of $Z=$ 0.004 the stellar yields computed with rotational velocities of $300 \mathrm{~km} \mathrm{~s}^{-1}$ or $400 \mathrm{~km} \mathrm{~s}^{-1}$ are the same (see their Table 5). For their lowest metallicity calculations, the yields computed with a velocity of $400 \mathrm{~km} \mathrm{~s}^{-1}$ are similar to those computed with $300 \mathrm{~km} \mathrm{~s}^{-1}$ for He, $\mathrm{C}$ and $\mathrm{O}$. Only for $\mathrm{N}$ there is a difference, namely the yield computed with $V_{\text {rot }}=400 \mathrm{~km} \mathrm{~s}^{-1}$ is a factor of two larger than the one computed with $V_{\text {rot }}=300 \mathrm{~km} \mathrm{~s}^{-1}$ for a $20 M_{\odot}$ and a factor of 3 larger for a $9 M_{\odot}$.

In Fig. 1 we compare the stellar yields for carbon and oxygen as computed by different authors, for the solar metallicity. For comparison we also show the yields of Maeder (1992) computed with strong mass loss and no rotation. It is clear from the figure (upper panel) that the yields of Maeder (1992 - open pentagons) for carbon are larger than those of MM even for the case of a large rotational velocity (filled squares). This difference arises mainly from the fact that in MM the mass loss rates are lower than those used in Maeder (1992). In particular, in MM the reduced mass loss rates accounting for the effects of clumping have been taken into account during the Wolf-Rayet phase. From Fig. 1, one sees that the WW and TNH yields multiplied by a factor of 3 in the $40-100 M_{\odot}$ mass range are similar to the new calculations of MM (see CMR2003). For oxygen (lower panel) the figure shows that the yields of Maeder (1992 - open pentagons) are larger than those of WW (triangles) or the ones given by MM. There is a good agreement among all the oxygen yields in the $15-25 M_{\odot}$ mass range.

In Fig. 2 we compare the predictions of MM (squares) for LIMS with those of $\mathrm{vdHG}$ (asterisks) for ${ }^{12} \mathrm{C}$, for different metallicities. It is clear from the figure that for all

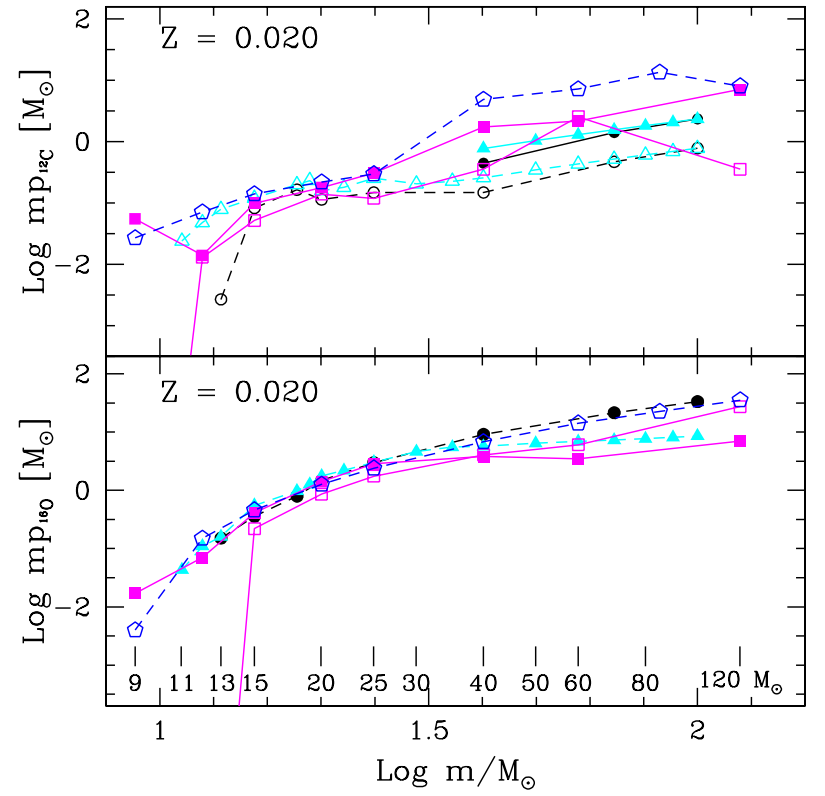

Fig. 1. Different stellar yields for ${ }^{12} \mathrm{C}$ (upper panel) and ${ }^{16} \mathrm{O}$ (lower panel) as functions of the initial stellar mass for massive stars, at solar metallicity: MM for $V_{\text {rot }}=0 \mathrm{~km} \mathrm{~s}^{-1}$ (open squares) and $V_{\text {rot }}=$ $300 \mathrm{~km} \mathrm{~s}^{-1}$ (filled squares); Thielemann et al. (1996) (circles); WW (triangles); Maeder (1992) (open pentagons). In the upper panel, the filled circles and filled triangles show the carbon yields of Thielemann et al. (1996) and WW, respectively, multiplied by a factor of 3 in the 40-100 $M_{\odot}$ mass range (see text).

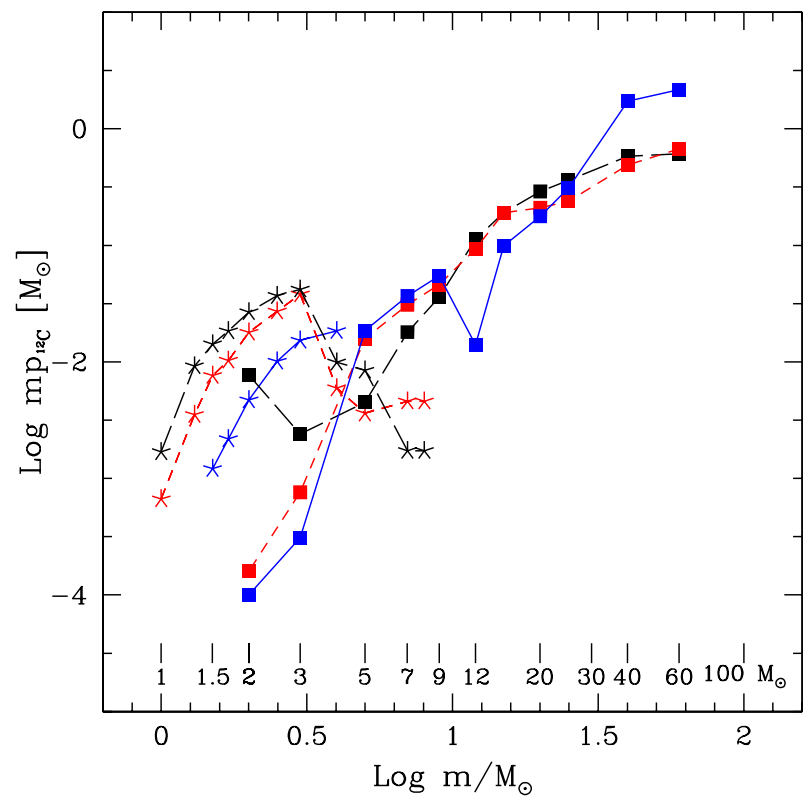

Fig. 2. Comparison of MM (squares) and vdHG (asterisks) yields of ${ }^{12} \mathrm{C}$ for 3 different values of metallicities (solid lines: solar, dashed line: $Z=0.004$ and long dashed line: $Z=0.001$ for $\mathrm{vdHG}$ and $Z=0.00001$ for MM), for masses up to $8 M_{\odot}$. Above $9 M_{\odot}$ we show the yields of MM for different metallicities.

metallicities, vdHG produce more carbon than MM. As discussed before this is mainly due to the fact that MM did not include the 3rd dredge up in their calculations and hence their yields for carbon, in this mass range, should be seen as lower limits. For $Z=10^{-5}$ (squares connected by a long-dashed 


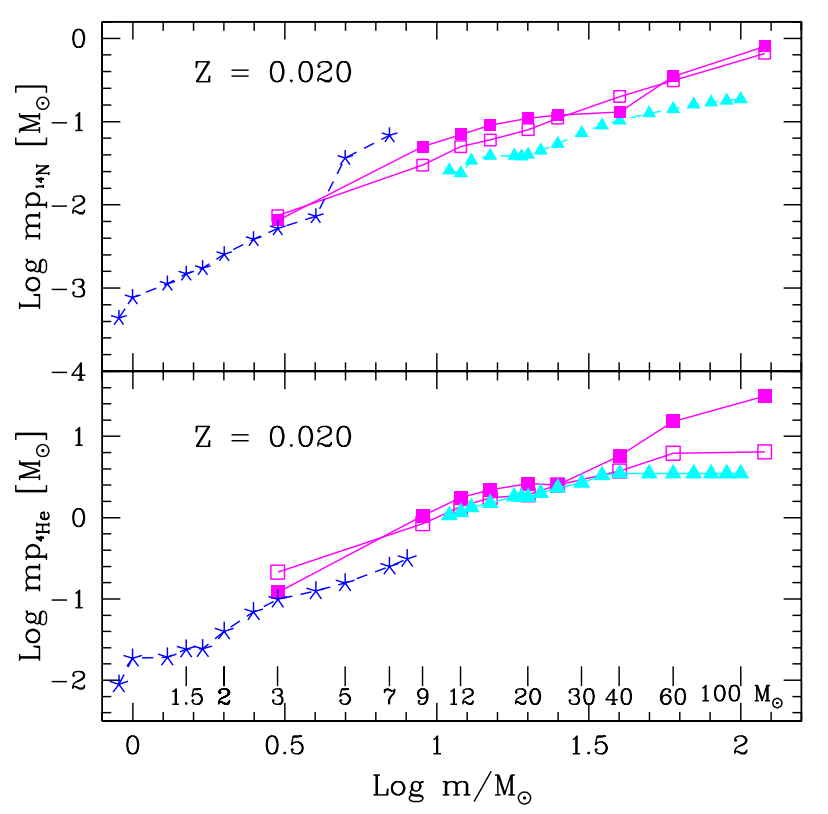

Fig. 3. Different stellar yields for ${ }^{14} \mathrm{~N}$ (upper panel) and ${ }^{4} \mathrm{He}$ (lower panel), for the whole stellar mass range. The symbols are as in Fig. 1. The asterisks represent the yields of van den Hoek \& Groenewegen (1997).

curve), MM do include the 3rd dredge up and this is why in this case more carbon is obtained. For the most massive stars the yields of MM predict more carbon at solar metallicities than in the two low metallicity cases. This is an effect of mass loss already present in Maeder (1992) calculations but which is less important in the new calculations of MM.

In Fig. 3 we compare the different stellar yields computed for ${ }^{14} \mathrm{~N}$ (upper panel) and ${ }^{4} \mathrm{He}$ (lower panel) for the whole mass range, for solar metallicities. For massive stars, mass loss by stellar winds, which is taken into account in the computation of MM, lead to larger yields of $\mathrm{N}$ and He relative to the work of WW (triangles). In the intermediate-mass range (5 to $8 M_{\odot}$ ) the nitrogen yields of vdHG (asterisks) are larger than the ones of MM and this is due to the contribution of the HBB. For the helium production, MM yields with rotation predict more helium than WW for massive stars, and also more than vdHG for intermediate mass stars. The consequence of the larger helium production when adopting the yields of $\mathrm{MM}$ on the $\Delta Y / \Delta Z$ ratio will be shown in Sect. 6.

In Fig. 4 we plot the yields of $\mathrm{MM}$ for ${ }^{14} \mathrm{~N}$, for different metallicities. Open symbols refer to stellar models were rotation is not taken into account, whereas filled squares refer to yields obtained from stellar models with $V_{\text {rot }}=300 \mathrm{~km} \mathrm{~s}^{-1}$. It is clear from the figure that rotation does not change much the nitrogen yields at solar metallicities (squares connected by a solid line), while it makes some difference at $Z=0.004$ (squares connected by short-dashed line). However, an important difference is seen at low metallicities (in this case $Z=10^{-5}$ - squares connected by long-dashed line). The yields with rotation of $\mathrm{MM}$ are similar to the ones of $\mathrm{vdHG}$ (for the case of $\eta_{\mathrm{AGB}}$ variable with metallicity), for all metallicities, for $m<4 M_{\odot}$, whereas, for masses between 4 and $8 M_{\odot}$, MM yields are

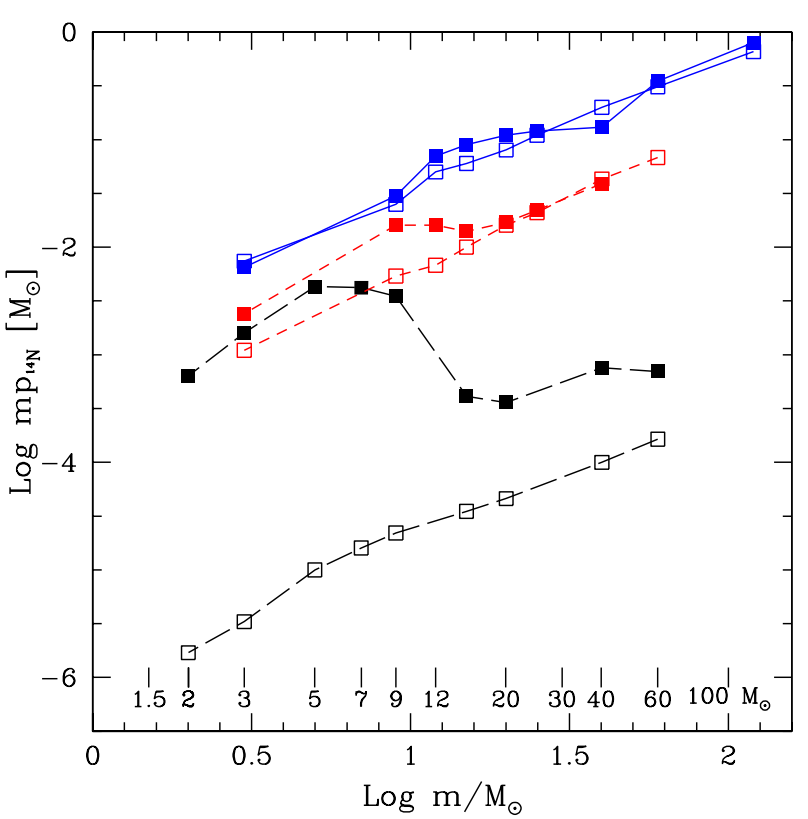

Fig. 4. MM stellar yields for ${ }^{14} \mathrm{~N}$, for the whole stellar mass range, for different metallicities. The symbols and lines are as in Fig. 2. The yields of MM for stellar models were rotation is not taken into account are also plotted (open squares).

substantially smaller. This is due to the strong HBB assumed in vdHG calculations.

\section{Chemical evolution model of the Milky Way}

In the next sections we show the results of chemical evolution models for the MW computed with the two different sets of yields discussed before. The model is that of CMR2003 (see also Chiappini et al. 2001, where a detailed description can be found). As explained in Sect. 1, the fundamental idea of this model is that the formation of the MW occurred in two different infall episodes, one forming the halo and part of the thick disk on a relative short timescale and another one forming the thin-disk on a longer timescale. In this model a threshold gas density is assumed and, as a consequence, the star formation rate becomes zero every time the gas density drops below the threshold value. The two-infall approach, combined with such a threshold, leads to a gap in the star formation before the formation of the thin-disk. During the "gap" in the star formation only elements produced by type Ia SNe and LIMS, born before the "gap", are restored into the ISM. As a consequence this model predicts an increase in the abundance ratios of elements restored on long-timescales over $\alpha$-elements (produced basically by massive short-lived stars) around a metallicity of $[\mathrm{Fe} / \mathrm{H}] \sim-0.6 \mathrm{dex}$ (which corresponds to the time of the halt in the SFR which we predict to be around 10 Gyrs ago - see Chiappini et al. 1997 for details).

\section{Carbon enrichment}

\subsection{The $[\mathrm{C} / \mathrm{Fe}]$ vs. $[\mathrm{Fe} / \mathrm{H}]$ diagram}

In Fig. 5 we show the variation of the $[\mathrm{C} / \mathrm{Fe}]$ ratio as a function of metallicity for stars in the MW compared with the 


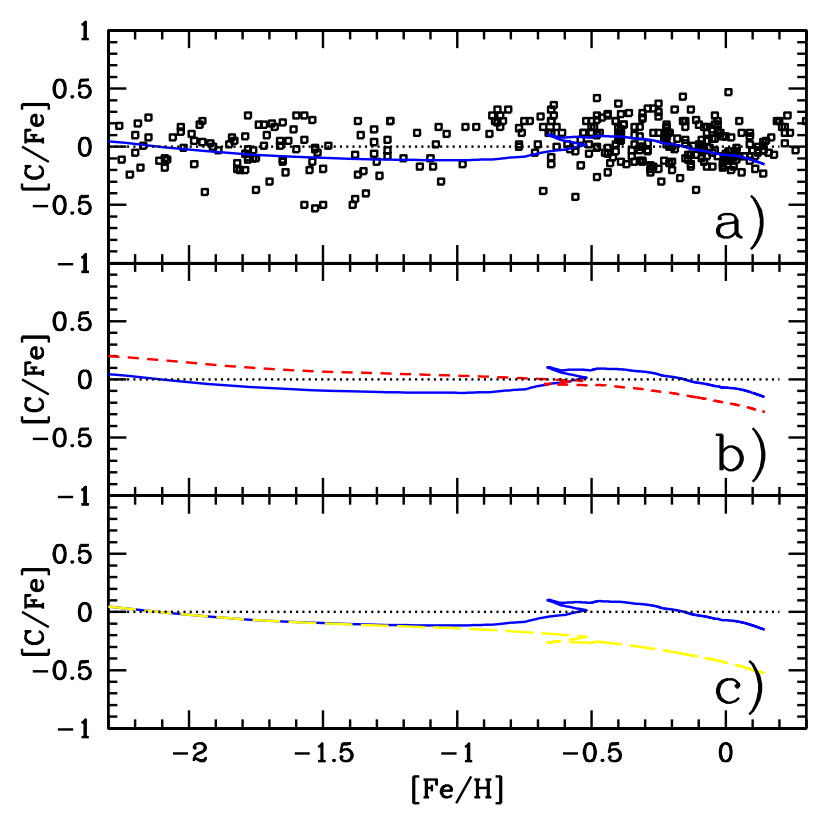

Fig. 5. $[\mathrm{C} / \mathrm{Fe}]$ vs. $[\mathrm{Fe} / \mathrm{H}]$. The dotted line in all panels markes the solar ratio. Panel a) shows a model computed with vdHG $+\mathrm{WW}$ yields (model A - solid line) compared to abundance data from the compilation of Chiappini et al. (1999). Both the model predictions and the abundance data show an almost solar $[\mathrm{C} / \mathrm{Fe}]$ ratio (down to $[\mathrm{Fe} / \mathrm{H}] \simeq-2$ ). In panel b) we compare the model A shown in a) with a model computed with MM yields (model B - short dashed line). Panel c) shows again the model A (solid line) compared with a similar model where the contribution from LIMS is suppressed (long-dashed line).

predictions of model A ( $\mathrm{vdHG}+\mathrm{WW}$ yields - solid line). The observed solar $[\mathrm{C} / \mathrm{Fe}]$ ratio from $[\mathrm{Fe} / \mathrm{H}] \sim-2$ up $[\mathrm{Fe} / \mathrm{H}]=0$ indicates that $\mathrm{C}$ and $\mathrm{Fe}$ are produced on similar timescales (CRM2003). This implies that the main producers of C cannot be massive stars unless the stellar yields vary strongly with metallicity. However, on the basis of the most up to date stellar models this latter alternative is unlikely and hence our conclusion is that the $\mathrm{C}$ we see today in the ISM comes mainly from LIMS, as originally suggested by Tinsley (1979). For metallicities below $[\mathrm{Fe} / \mathrm{H}] \sim-2$, type Ia and intermediate-mass stars did not have yet time to contribute to the enrichment and the behavior of $[\mathrm{C} / \mathrm{Fe}]$ can be substantially different as it will depend essentially on the variation of the ejected masses of $\mathrm{C} / \mathrm{Fe}$ from massive stars.

In Fig. $5 b$ we compare the $[\mathrm{C} / \mathrm{Fe}]$ predicted by model $\mathrm{A}$ (solid line) and model B (computed with MM stellar yields short-dashed line). Model B results are good for low metallicities, but predicts too few carbon during the thin disk phase $([\mathrm{Fe} / \mathrm{H}]>-0.8)$. The main reason for that is the absence of the 3rd dredge up in MM models for metallicities larger than $Z=10^{-5}$. In fact, as shown in Fig. 5c, a similar behavior is seen if in model A we "turn off" the contribution to C from LIMS (long-dashed line curve).

\subsection{The $\log (\mathrm{C} / \mathrm{O})$ vs. $\log (\mathrm{O} / \mathrm{H})$ diagram}

\subsubsection{Abundance data}

Several papers devoted to the discussion of the $\log (\mathrm{C} / \mathrm{O}) \mathrm{vs}$. $\log (\mathrm{O} / \mathrm{H})$ relation and its interpretation, consider the abundance data of different galaxies together with the abundance data of the MW (e.g. Carigi 2000; Henry et al. 2000). As discussed in CRM2003 the abundance data for the MW contain an important temporal information in the sense that low metallicity main sequence stars represent the composition of the ISM at the time of their formation. This is not the case if we plot together the present time abundances in HII regions in other galaxies. In addition, each galaxy has suffered a particular enrichment history. Given this fact we decide to plot here only the stellar abundances of $\mathrm{C}$ and $\mathrm{O}$ observed in the MW. Moreover, since our last paper (CRM2003) a new homogeneous data sample was published by Nissen (2003) which include halo, thick disk and thin disk stars. This data set shows a very important new feature (see Fig. 6): it seems to indicate a discontinuity between the halo and thin disk stars. Moreover, this discontinuity seems to happen exactly at the same metallicity where Gratton et al. (1996) and Fuhrmann (1998) observed a lack of stars in $\mathrm{Fe} / \mathrm{O}$ and $\mathrm{Fe} / \mathrm{Mg}$ plots, respectively. More data are necessary to confirm this possible gap in the stars.

\subsubsection{Model predictions}

In Fig. 6 (upper left panel) we compare the data mentioned before with our model A prediction (solid line). As it can be seen, a model in which a halt in the star formation is predicted and including stellar yields where an important amount of $\mathrm{C}$ comes from LIMS, produces a discontinuity in the $\log (\mathrm{C} / \mathrm{O})$ vs. $\log (\mathrm{O} / \mathrm{H})$ diagram, similar to what is observed. This would not be the case if $\mathrm{C}$ were mainly produced from massive stars. In fact, in the same figure (upper right panel) we show model A but with no contribution from LIMS to carbon. In this case, as expected, we obtain a flat $\log (\mathrm{C} / \mathrm{O})$ along the whole metallicity range.

Figure 6 (lower left panel) shows the prediction of model B (computed with MM yields). Two things can be noted: a) the present $\log (\mathrm{C} / \mathrm{O})$ is too low and b) the discontinuity discussed before is not present anymore. In fact, since in this case the LIMS do not produce much $\mathrm{C}$ (due to the lack of the 3rd dredge-up), we see essentially what would be expected for the ratio of two elements produced by massive stars namely, during the star formation "gap" both elements stop being produced, and the only effect seen is the decrease in the $\mathrm{O} / \mathrm{H}$ ratio due to the second infall that forms the disk (assumed to be primordial) which produces the "looping" seen in the figure. In the low right panel we show the effect of assuming instantaneous recycling approximation (I.R.A.) when trying to interpret the $\log (\mathrm{C} / \mathrm{O})$ abundance ratio. As $\mathrm{C}$ is restored into the ISM on longer timescales than O, the I.R.A. is not correct in this case. We illustrate this point by plotting the integrated yields of MM (squares) computed for different metallicities and integrated over different mass ranges (indicated in the figure in brackets), assuming a Scalo IMF, as in the chemical evolution 


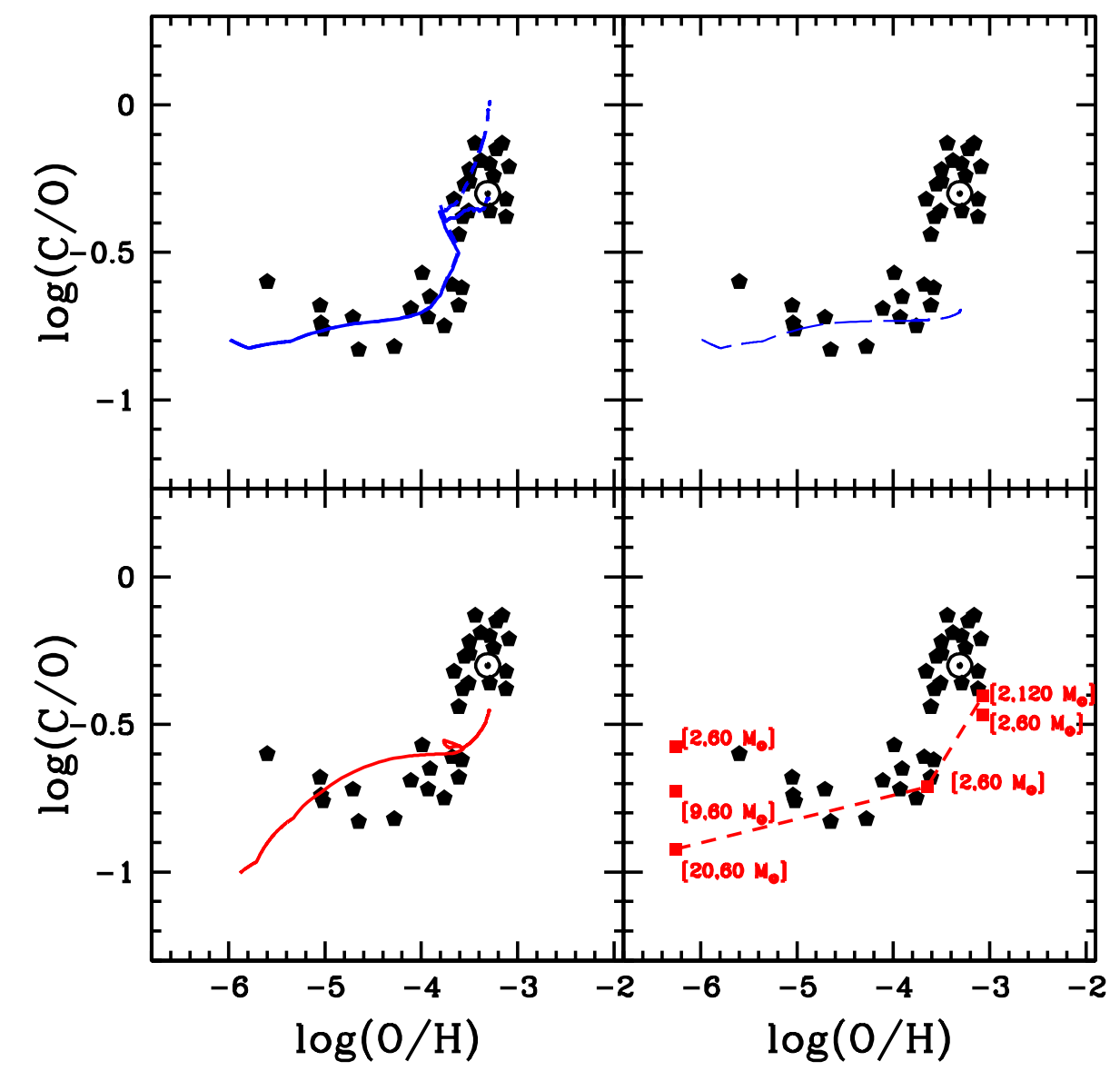

Fig. 6. Model predictions for the galactic evolution of the $\mathrm{C} / \mathrm{O}$ ratio as a function of $\mathrm{O} / \mathrm{H}$ compared with data obtained by Nissen (2003). The abundance data include only stars in the Milky Way (halo, thick disk and thin disk stars) whose abundances are representative of the ISM from which they formed. The solar value from Allende-Prieto et al. $(2001,2002)$ is also shown. In the left upper panel a chemical evolution model for the MW computed with vdHG + WW yields (model A) is shown (solid line). The dashed line shows the same model computed with stellar yields for LIMS given in the table for $Z=0.001$ from vdHG with $\eta_{\mathrm{AGB}}=1$ (an upper limit for the C production in LIMS). In the right upper panel we show again model A but with no contribution from LIMS. In the lower left panel model B prediction is shown (computed with stellar yields including rotation effects from Meynet \& Maeder 2002). In the lower right panel we plot directly the integrated yields (for different ranges of masses - squares) at different metallicities (without the use of a chemical evolution model). This is a simplistic approach that implicitly assumes instantaneous recycling approximation and thus neglects the effects due to stellar lifetimes.

models presented here. As we can see, the $\log (\mathrm{C} / \mathrm{O})$ at solar metallicity, obtained by integrating the stellar yields, is slightly larger than what is obtained by using the same stellar yields in a chemical evolution model which takes into account the stellar lifetimes. In fact, the final $\mathrm{C} / \mathrm{O}$ value achieved by model $\mathrm{B}$ (in the lower left panel) is lower than that obtained when assuming I.R.A. (right lower panel). Because of the relaxation of I.R.A. in our models, the low mass stars formed out of gas enriched in oxygen relative to iron or carbon, at early times, restore their pristine gas with $\mathrm{C} / \mathrm{O}$ ratios characteristic of the halo phase, thus lowering the present $\mathrm{C} / \mathrm{O}$ ratio.

In summary, the contribution of LIMS to the abundance of $\mathrm{C}$ is important. The opposite conclusion was suggested by some authors and arised for two reasons: a) the difficulty of reproducing the $\mathrm{C} / \mathrm{O}$ ratio versus $\mathrm{O} / \mathrm{H}$ at large $\mathrm{O}$ abundances and b) the yields of Maeder (1992), where a strong mass loss, operating in stars of high metal content, allows the most massive stars to loose more and more carbon as a function of stellar mass and metallicity. This solution creates some difficulty then in explaining the $[\mathrm{C} / \mathrm{Fe}]$ vs. $[\mathrm{Fe} / \mathrm{H}]$ running flat for all metallicities (as shown in CMR2003). Moreover, as shown in this section, the new yields of MM do not predict anymore a rise in the $\mathrm{C} / \mathrm{O}$ ratio as a function of oxygen of the same magnitude as it would be obtained with Maeder (1992) yields. Our results show that the solar $\mathrm{C} / \mathrm{O}$ value can be explained by models which predict a break in the star formation rate before the thin disk formation together with stellar yields for intermediate mass stars including the 3 rd dredge up.

\section{The $\mathrm{N}$ enrichment}

\subsection{The Milky Way}

Ideally the best way to ascertain the nature of nitrogen would be to look at the $\mathrm{N}$ abundances in the stars in the MW since they represent a true evolutionary sequence, where the stars with lower metallicity are the oldest ones. Unfortunately, the $\mathrm{N}$ abundances for stars in the MW are still uncertain, especially at low metallicities (see Nissen 2003). Current data show a $[\mathrm{N} / \mathrm{Fe}]$ ratio which is below solar at low metallicities and then 


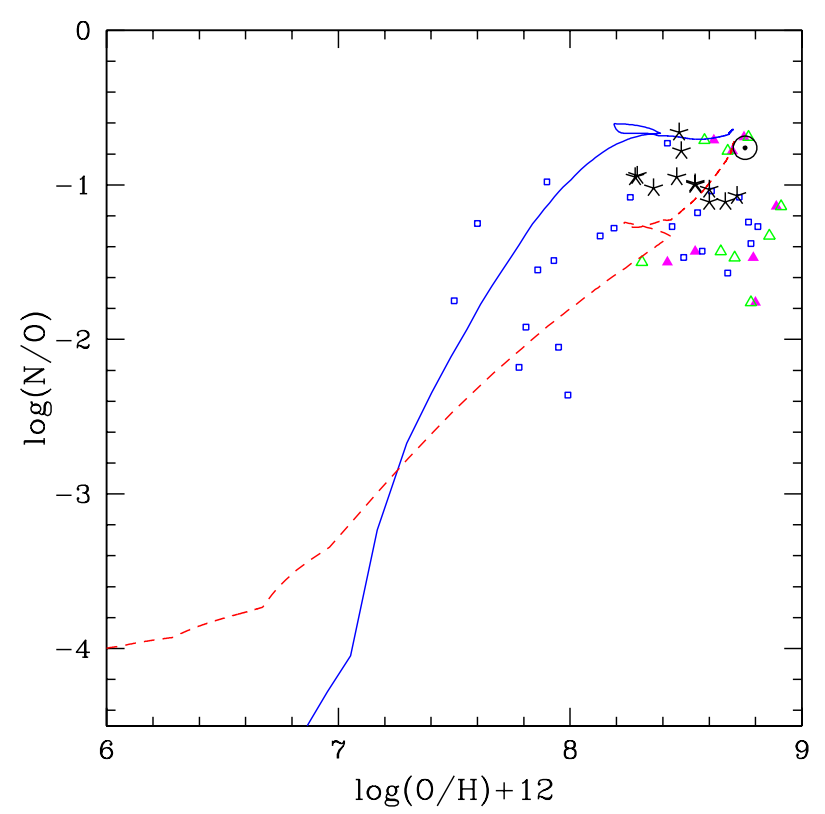

Fig. 7. Diagram showing $\log (\mathrm{N} / \mathrm{O})$ vs. $\log (\mathrm{O} / \mathrm{H})+12$ for the Milky Way (model A: solid curve, and model B: dashed curve; see CRM2003 for a description of the abundance data).

increases and flattens in disk stars (see CRM2003). This behavior agrees with the assumption that primary $\mathrm{N}$ production in massive stars is small. Here we show that this is still true even when the yields of MM, which predict some primary N contribution from massive stars, are adopted.

In Fig. 7 we show the predictions of models $\mathrm{A}$ (solid line) and $\mathrm{B}$ (dashed line) for $\log (\mathrm{N} / \mathrm{O})$ vs. $\log (\mathrm{O} / \mathrm{H})+12$ for the solar vicinity. The data points are from a compilation by CRM2003. It can be seen that the primary $\mathrm{N}$ production in massive stars predicted by model $\mathrm{B}$, with the MM yields, is small and gives rise to a sort of "plateau" at $\log (\mathrm{N} / \mathrm{O}) \sim-4$ and $\log (\mathrm{O} / \mathrm{H})+12 \leq 7$. In fact, almost the same value is obtained by MM when the stellar yields corresponding to $\log (\mathrm{O} / \mathrm{H})+12=5.74$ and velocities of $300 \mathrm{~km} \mathrm{~s}^{-1}$, are integrated in the $20-120 M_{\odot}$ mass range, which should be the stars contributing to the ISM enrichment at such low metallicities in the case of the MW (see Fig. 8). This is the first result, namely that the primary $\mathrm{N}$ contribution from massive stars predicted by $\mathrm{MM}$ is small. A second result is that the increase of $\mathrm{N}$ in intermediate mass stars in model B is more gentle than in model A where the HBB contributes a substantial amount primary $\mathrm{N}$. Models A and B seem to agree with the available data for the MW but more data are clearly needed, especially at low metallicities.

The above results show that a plateau, due to primary $\mathrm{N}$ production in massive stars as suggested by $\mathrm{MM}$ yields, occurs at a $\log (\mathrm{N} / \mathrm{O})$ ratio which is far below $(\log (\mathrm{N} / \mathrm{O}) \sim$ -4.0 dex when the yields of MM for $V_{\text {rot }}=300 \mathrm{~km} \mathrm{~s}^{-1}$ are adopted) the "plateau" seen either in blue compact galaxies (BCGs) at a $\log (\mathrm{N} / \mathrm{O}) \sim-1.6 \mathrm{dex}$ (Izotov \& Thuan 1999) or in some DLAs at a $\log (\mathrm{N} / \mathrm{O}) \sim-2.2 \mathrm{dex}$ (Prochaska et al. 2002). Only when very high rotation velocities are considered, the primary $\mathrm{N}$ production by massive stars may become

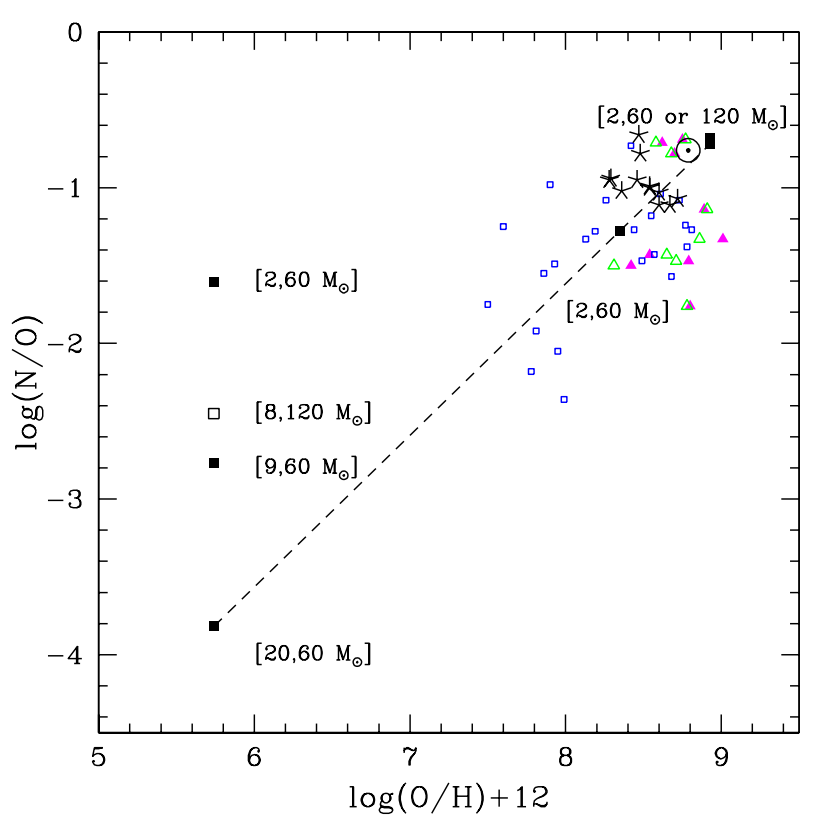

Fig. 8. Diagram showing $\log (\mathrm{N} / \mathrm{O})$ vs. $\log (\mathrm{O} / \mathrm{H})+12$ obtained by integrating the MM yields in different mass ranges as indicated in brackets (filled squares). As it can be seen by comparing with Fig. 7 the main stars contributing at metallicities of the order of $\log (\mathrm{O} / \mathrm{H})+12 \sim$ 5.8 are those above $\sim 20 M_{\odot}$. The empty square refers to the case with rotational velocity of $400 \mathrm{~km} \mathrm{~s}^{-1}$.

significant (see Fig. 8 the empty square which corresponds to $\left.V_{\text {rot }}=400 \mathrm{~km} \mathrm{~s}^{-1}\right)$.

Izotov \& Thuan (1999) have claimed that BCGs show a flat $\log (\mathrm{N} / \mathrm{O})$ plateau at low metallicities and suggested it to represent the minimum $\mathrm{N} / \mathrm{O}$ value due to the primary $\mathrm{N}$ production in massive stars. As discussed in CRM2003 we think this interpretation is not correct since in the case of BCGs we are comparing different objects at present time and the observed $\mathrm{N} / \mathrm{O}$ ratios are therefore the products of 13 Gyrs of evolution. For BCGs this diagram is not the same as for the MW stars, in the sense that the $x$-axis $(\log (\mathrm{O} / \mathrm{H})+12)$ cannot be interpreted as a time axis. Moreover, if that was the case a plateau around $\log (\mathrm{N} / \mathrm{O}) \sim-1.6$ should also be seen for the MW stars. Current data for the MW do not support this idea (CRM2003). However, the models shown in Fig. 7 apply only to the solar vicinity. In what follows we would like to better assess the impact of the MM yields on the interpretation of BCGs and DLAs abundance data by computing chemical evolution models which apply to these systems.

\subsection{Blue compact galaxies and DLAs}

Blue compact galaxies are best reproduced by short and moderately intense bursts of star formation followed by longer quiescent periods (Matteucci \& Chiosi 1983). As shown in CRM2003, the large spread observed both in the N/O versus $\mathrm{O} / \mathrm{H}$ and $\mathrm{C} / \mathrm{O}$ versus $\mathrm{O} / \mathrm{H}$ diagrams for oxygen abundances larger than 7.6 can be explained as arising from different chemical evolution histories of different galaxies (i.e. different star formation efficiencies, different burst ages and different burst durations). The nature of DLAs is still a matter of debate but their chemical characteristics seem to be in agreement with the 


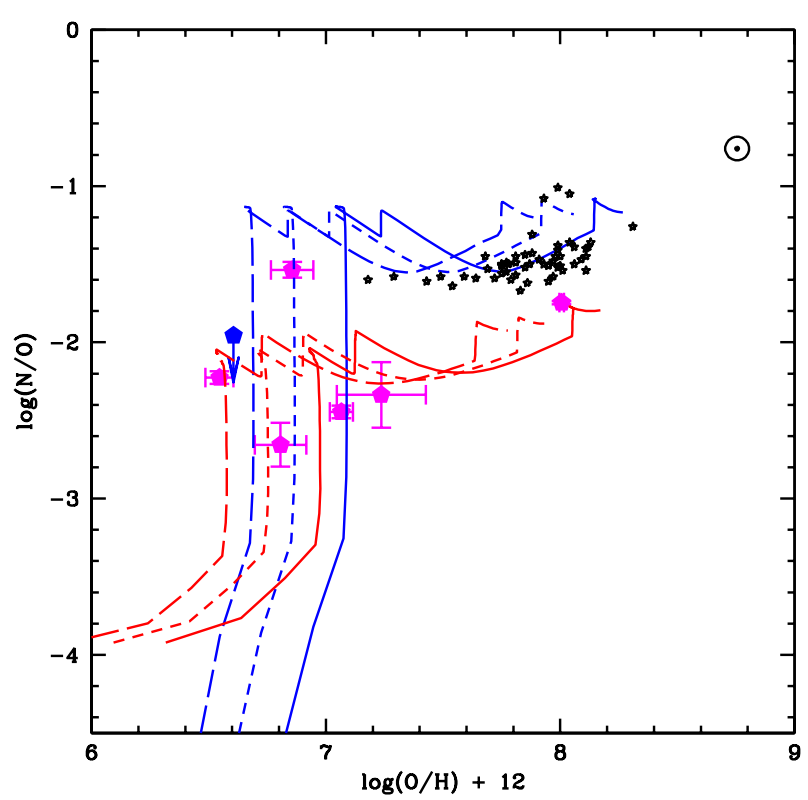

Fig. 9. The same diagram as in Fig. 6 but now for dwarf galaxies of Izotov \& Thuan (1999) and DLAs from Centurion et al. (2003a) (filled pentagons - with a new determination from Centurion et al. 2003b). Model predictions are explained in the text. For the lower family of curves an abundance ratio $\log (\mathrm{N} / \mathrm{O}) \sim-2.5$ is attained after $53 \mathrm{Myr}$ from the start of the star formation, while for the upper curves $\log (\mathrm{N} / \mathrm{O})=-1.8$ after $53 \mathrm{Myr}$.

idea that they represent either the progenitors of the current dwarf galaxies or the outer parts of disk galaxies (see Prantzos \& Boissier 2000; Lanfranchi \& Matteucci 2003; Calura et al. 2003). In particular, Lanfranchi \& Matteucci (2003) suggest that some of the DLAs chemical properties can be well fitted by models for BCGs, with roughly 4 bursts of star formation, with star formation efficiencies in the range of $0.1-0.9 \mathrm{Gyr}^{-1}$. This kind of models are able to fit the $[\alpha / \mathrm{Fe}]$ observed in DLAs but still predict too much $\mathrm{N}$ when the yields of $\mathrm{vdHG}$ are adopted.

In Fig. 9 we show the same kind of diagram as in Fig. 7 but now we plot the DLA abundance data (from Centurion et al. 2003a,b-pentagons) for objects where oxygen was measured or a limit was given. Also shown are the BCGs observed by Izotov \& Thuan (1999) (small symbols). The curves show "bursting" models computed with four bursts and a Salpeter $\mathrm{IMF}^{2}$. In these models, similar to Lanfranchi \& Matteucci (2003), we assumed 4 bursts at $t=1,10,13$ and $13.98 \mathrm{Gyrs}$ with a duration of $0.02,0.01,0.2$ and 0.02 Gyrs, respectively. The "family" of upper curves shows models computed with different star formation efficiencies $\left(0.1,0.2\right.$ and $0.3 \mathrm{Gyr}^{-1}$ shown as long-dashed, short-dashed and solid lines, respectively) and with the same nucleosynthesis prescriptions of model A (i.e. $\mathrm{vdHG}+\mathrm{WW}$ ), whereas the lower curves show the same models computed with the yields of MM. In this case the DLAs $\mathrm{N}$ abundance can be well explained.

In fact, given the more gentle increase of the $\mathrm{N}$ when the yields of MM are adopted (due to less N from LIMS) it is

${ }^{2}$ In CRM2003 we suggest that to be able to fit the N/O ratio observed in BCGs a flatter IMF was necessary, but Lanfranchi \& Matteucci (2003) concluded that such models would predict $[\mathrm{O} / \mathrm{Fe}]$ ratios larger than the observed ones.

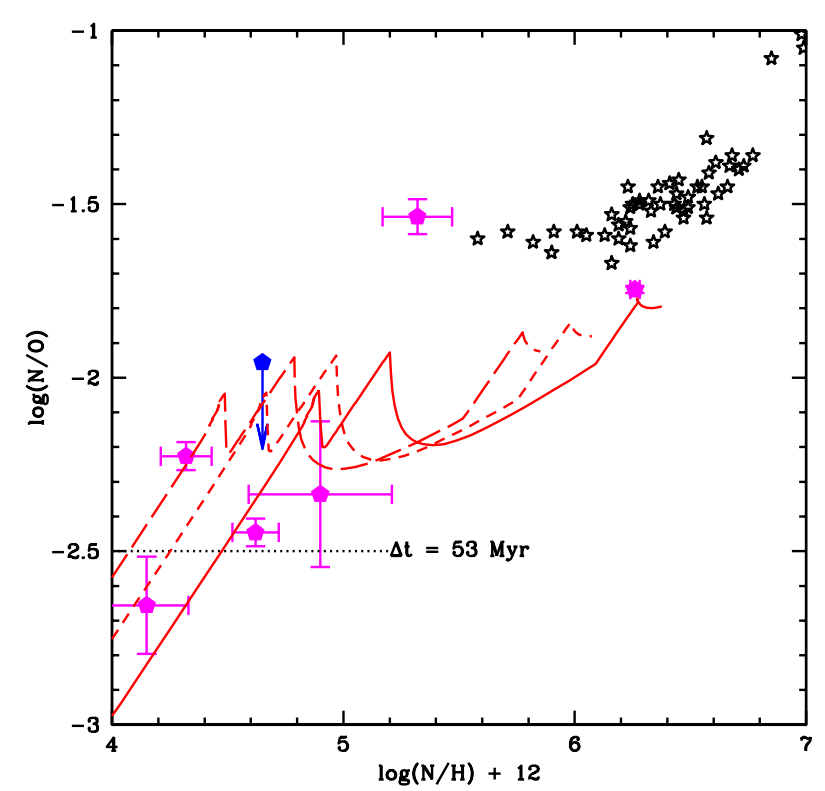

Fig. 10. $\log (\mathrm{N} / \mathrm{O})$ vs. $\log (\mathrm{N} / \mathrm{H})+12$ diagram. Dwarf galaxies are from of Izotov \& Thuan (1999 - black dots); DLAs from Centurion et al. (2003a,b) (filled hexagons). The models are the same shown in Fig. 9 (lower family of curves, with MM yields). The $\Delta t=53 \mathrm{Myr}$ indicates the time elapsed from the beginning of star formation and the dotted line represents an isochrone. In other words, the objects on that line have the same age but different star formation efficiency.

possible to obtain a low $\log (\mathrm{N} / \mathrm{O})$ without predicting a too high $[\alpha / \mathrm{Fe}]$ enhancement, as required by observations in DLAs. When the MM yields are adopted, a $\log (\mathrm{N} / \mathrm{O})$ ratio of -2.5 is achieved after roughly 53 Myrs (see Fig. 10). This means that intermediate mass stars are already contributing for the $\mathrm{N}$ enrichment even in DLAs which show a mean $\log (\mathrm{N} / \mathrm{O}) \sim$ -2.2 dex (we recall that the lifetime of an $8 M_{\odot}$ is of the order of $30 \mathrm{Myr}$ ). Models with vdHG yields can also explain the DLA abundances, but in this case DLAs with a low N/O ratio would be very young systems and this may happen as an odd coincidence.

In Figs. 10 and 11 we plot $\log (\mathrm{N} / \mathrm{O})$ vs. $\log (\mathrm{N} / \mathrm{H})+12$. The models shown in Fig. 10 are the same as the lower $\log (\mathrm{N} / \mathrm{O})$ "family" of models of Fig. 9 ("bursting models") computed with MM yields. In Fig. 11 we show a prediction for an outer region of the MW disk (in this case $16 \mathrm{kpc}$ ): the chemical evolution of the outer regions of the Milky Way disk are computed by assuming an inside-out formation of the disk, thus having a lower star formation rate at any time than the solar vicinity and resembling the evolution of magellanic irregular galaxies (the model shown here was computed with the same parameters as model A of Chiappini et al. 2001; see also Calura et al. 2003). These two figures suggest: a) both "bursting" models and outer disks can explain the measured abundance ratios in DLAs; b) in "bursting models" the low values of N/O will last for many Gyrs while in outer regions of spiral disks the increase of $\mathrm{N}$ is faster (in Fig. 11 we indicate the elapsed time since the start of the $\mathrm{SF}$ at the corresponding $\mathrm{N} / \mathrm{O}$ value). For the models in Fig. 10 the $\mathrm{N}$ enrichment is slower. By instance, for the short dashed curve, which represents a model computed with an intermediate value for the star formation ef- 


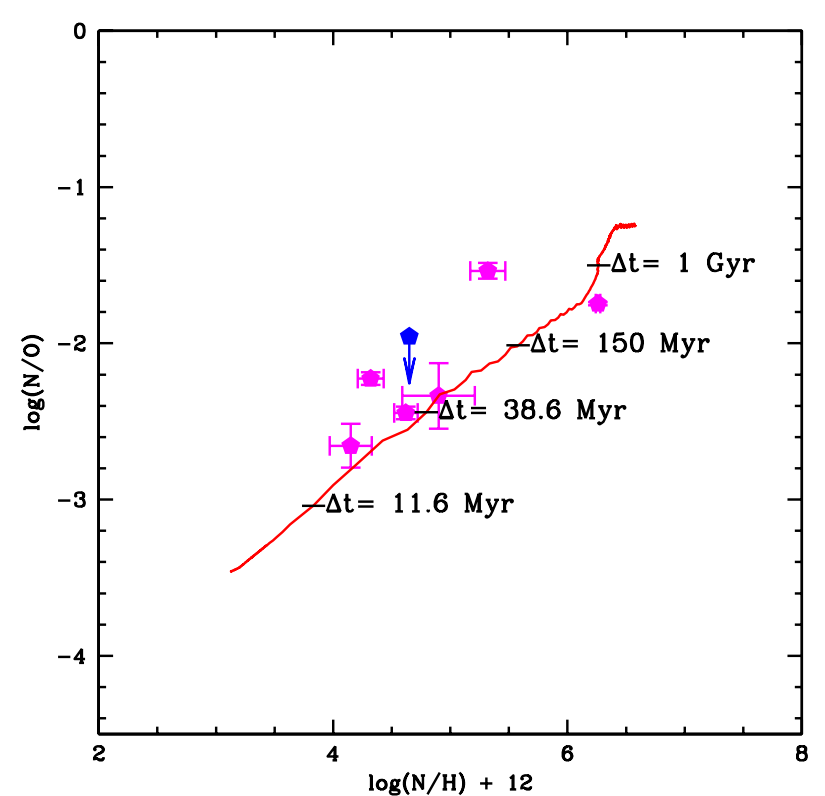

Fig. 11. $\log (\mathrm{N} / \mathrm{O})$ vs. $\log (\mathrm{N} / \mathrm{H})+12$ diagram. DLAs from Centurion et al. (2003a,b) (filled hexagons). Model prediction for MW (with MM yields) at an outer region $-16 \mathrm{kpc}$ in this example. The $\Delta t$ s indicate the time elapsed from the beginning of star formation, in other words the various galactic ages.

ficiency $\left(0.2 \mathrm{Gyr}^{-1}\right)$, we have that after roughly $35 \mathrm{Myrs}$ the system reaches a $\log (\mathrm{N} / \mathrm{O})$ of -2.7 . After that, $\log (\mathrm{N} / \mathrm{O})$ increases slowly achieving the first peak only after $\sim 3$ Gyrs due to the low star formation efficiency and also to the fact that the first burst formed not many stars due to its short duration. This value stays almost constant until the second burst occurs then lowering $\log (\mathrm{N} / \mathrm{O})$ again, in this case after 9 Gyrs from the start of the star formation. This means that for most of the time these systems will show a low $\log (\mathrm{N} / \mathrm{O})$ and also an $[\mathrm{O} / \mathrm{Fe}] \mathrm{ra}-$ tio around 0.3-0.2 dex in agreement with what is observed in DLAs ${ }^{3}$. Our results suggest that DLAs could be explained by systems which are similar to BCGs, with short duration bursts. In this framework, even the "low N/O group of DLAs" (first identified by Prochaska et al. 2002) can be explained as systems in which both massive stars and LIMS are contributing to the ISM enrichment. It remains to be seen to what extent the MM yields for $\mathrm{N}$ in LIMS will be increased if the HBB would be taken into account.

In the scenario of Lanfranchi \& Matteucci (2003) or Calura et al. (2003), DLAs with low $\log (\mathrm{N} / \mathrm{O})$ are young systems, i.e., systems where the $\mathrm{N}$ coming from intermediate mass stars did not have time yet to contribute to the ISM enrichment. This is a consequence of the fact that in both works the authors adopted vdHG yields. On the other hand, in the "bursting" models presented here, computed with MM yields, the so called "low $\log (\mathrm{N} / \mathrm{O})$ DLAs" are systems spanning a broad range of ages, as in this case the increase of $\mathrm{N}$ from

${ }_{3}$ This value is based essentially on $\mathrm{O} / \mathrm{Zn}$ and $\mathrm{Si} / \mathrm{Zn}$ abundance ratios. In fact, part of Fe in DLAs is likely to be in dust form, while $\mathrm{O}$ and $\mathrm{Zn}$ are not depleted in dust. For Si only a mild depletion is expected (see discussions in Centurion et al. 2003a,b; Pettini et al. 2002; Vladilo 2002).

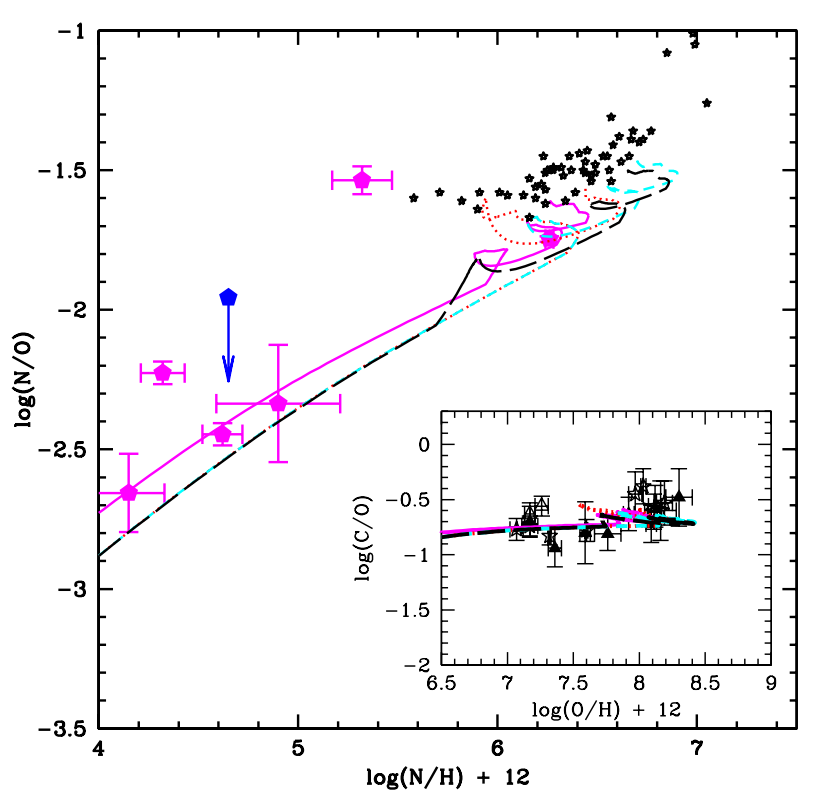

Fig. 12. Same as Fig. 10 but now we show model predictions for BCGs (see text). Also shown is a $\log (\mathrm{C} / \mathrm{O})$ vs. $(\mathrm{O} / \mathrm{H})$ plot. Details on the abundance data can be find in CRM2003.

LIMS is slow. However, not only we still have to explain DLAs systems with $\log (\mathrm{N} / \mathrm{O})$ ratios similar to the ones observed in BCGs $(\log (\mathrm{N} / \mathrm{O}) \sim-1.6 \mathrm{dex})$, but we also have to explain the BCGs themselves.

This can be obtained with the same kind of models but assuming that, in these systems, the initial burst of SF involved more mass or, in other words, lasted longer. The star formation efficiencies are of the same order than in the models we used before to explain the "low $\log (\mathrm{N} / \mathrm{O})$ DLAs" (larger values would produce produce too much $\mathrm{O} / \mathrm{H}$ or $\mathrm{N} / \mathrm{H}$ ). To illustrate this point we show in Fig. 12 a family of models similar to the ones shown in Fig. 10, computed with MM, but in which the bursts of star formation lasted longer $(0.8 \mathrm{Gyrs})$. In this case it is clear that the larger $\log (\mathrm{N} / \mathrm{O})$ observed in BCGs can be achieved. By varying parameters such as the burst duration and the number of bursts we can thus explain the larger $\log (\mathrm{N} / \mathrm{O})$ observed in BCGs and in some DLAs. With a longer burst, both the oxygen and the N/O ratio increase. The relatively higher $\mathrm{N}$ is due to the fact that the star formation lasts longer and thus the secondary $\mathrm{N}$ contribution is larger. This figure shows that these models are also able to reproduce the observed $\mathrm{C} / \mathrm{O}$ ratios in BCGs.

In summary, our results show that once we adopt the MM yields, the low $\log (\mathrm{N} / \mathrm{O})$ in DLAs can be explained by "bursting models" of the kind of the ones suggested by Lanfranchi \& Matteucci (2003). DLAs with low $\log$ (N/O) ratios would be systems similar to BCGs but in which the bursts of star formation were brief. In such models the $\mathrm{N}$ comes not only from massive stars but also from LIMS. Models with longer star formation bursts can explain the abundance ratios of BCGs and DLAs with larger $\log (\mathrm{N} / \mathrm{O})$ ratios. Notice that in this framework, the "low" and "high" $\log (\mathrm{N} / \mathrm{O})$ DLAs would not have an age difference but rather a difference in their star formation mode. Moreover, the difference between these two 
kind of systems will be better seen in a $\log (\mathrm{N} / \mathrm{O})$ vs. $\log (\mathrm{N} / \mathrm{H})$ diagram as suggested by Centurion et al. (2003a). However, at variance with what is suggested by these authors, we favor the view that the discontinuity seen in such a diagram indicates a difference in the star formation rather than in the age. Our conclusions depend on the adopted stellar yields and can change if either the amount of primary $\mathrm{N}$ in massive stars is larger than the one adopted here or if HBB contributes to an important amount of $\mathrm{N}$ in LIMS.

An anti-correlation between N/O and O/Fe would certainly help us to clarify which is the best scenario for the formation of DLAs. In the scenario in which DLAs with low $\log (\mathrm{N} / \mathrm{O})$ are young systems, one would expect these objects to show larger $\mathrm{O} / \mathrm{Fe}$ ratios compared to systems with $\log (\mathrm{N} / \mathrm{O}) \sim-1.6$ (as the BCGs). However, this interpretation is complicated by the fact that $\mathrm{Fe}$ is depleted onto dust grains. In fact, accounting for the fraction of $\mathrm{Fe}$ in dust form requires taking into consideration the full complement of abundances measurements in a DLA (see Vladilo 2002; Pettini et al. 2002). Another discriminant would be s-process elements produced uniquely by intermediate mass stars (heavy s-elements).

\section{The helium enrichment}

Finally, in this last section we compare our results for the evolution of helium in the MW predicted by models A and B. As shown in Sect. 2 the MM yields for helium are larger than the ones of vdHG and WW. In this work we decided to adopt the primordial composition from Chiappini et al. (2001), where for the pregalactic helium abundance we had $Y_{\mathrm{p}}=0.241$. In Fig. 13 we show the predictions of models A (solid line) and B (dashed line) for the evolution of the helium abundance as a function of metallicity (oxygen in this case). As it can be seen, model B is in better agreement with the solar value. This model predicts a solar value of $Y_{\odot}=0.265$ and a value of 0.272 at the present time. Model A instead predicts $Y_{\odot}=0.253$ and $Y=0.257$ at the present time. As already shown in Chiappini et al. (2002), when the yields of $\mathrm{vdHG}+\mathrm{WW}$ are adopted the models are marginaly consistent with the solar value. Moreover, while models adopting vdHG+WW yields predict a $\Delta Y / \Delta Z$ value around 1.5, model B (with MM yields) predicts $\Delta Y / \Delta Z \sim 2.4$. This larger value for $\Delta Y / \Delta Z$ seems to be in better agreeement with the observations (see Pagel 2000).

However, as shown in Chiappini et al. 2002, by means of our chemical evolution model for the MW, we can constrain the primordial value of deuterium abundance and we find a value of $(\mathrm{D} / \mathrm{H})_{\mathrm{p}} \leq 4 \times 10^{-5}$, which implies $Y_{\mathrm{p}}>0.244$. This is in fact in agreement with the recent results from WMAP which implies $Y_{\mathrm{p}}=0.248$ (see Romano et al. 2003). In Fig. 13 we also show a model computed with the same nucleosynthesis prescriptions of model $\mathrm{B}$, but assuming $Y_{\mathrm{p}}=0.248$ (dotted line). In this case we obtain $Y_{\odot}=0.272$ in agreement with Grevesse \& Sauval (1998) and Bahcall et al. (2001). Model A computed with $Y_{\mathrm{p}}=0.248$ gives $Y_{\odot}=0.261$ and it is still only marginally consistent with the solar value.

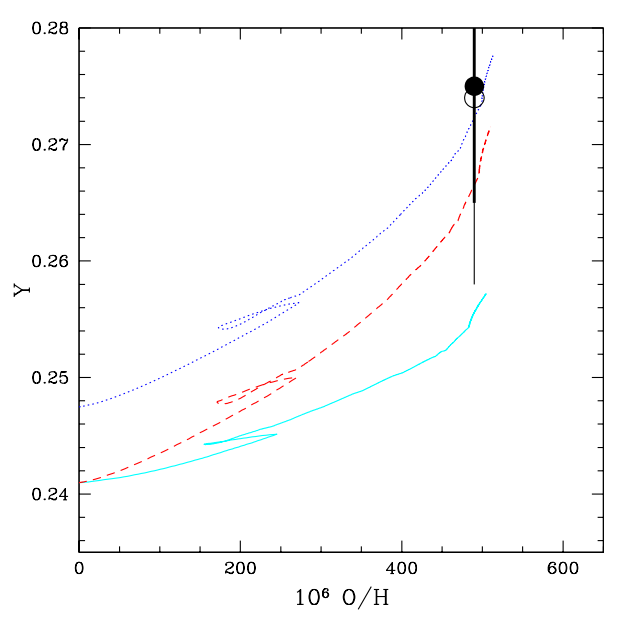

Fig. 13. $Y$ vs. $10^{6} \mathrm{O} / \mathrm{H}$ predicted by model A (solid line) and B (dashed line). The dotted-line shows a model which is the same as model B but computed with $Y_{\mathrm{p}}=0.248$ (see text). The solar value is also shown (where oxygen is from Allende-Prieto et al. 2001 and $Y$ is from Anders \& Grevesse 1989 - open circle with thin errorbar and Grevesse \& Sauval 1998 - black circle with thick errorbar).

\section{Conclusions}

- We suggest, on the basis of the available data in the solar vicinity, that $\mathrm{C}$ should come mainly from low- and intermediate-mass stars. This is at variance with the interpretation by several authors (e.g. Carigi 2000; Henry et al. 2000 ) that $C$ should originate mainly in massive stars. This conclusion was based on the yields of Maeder (1992) predicting a strong metallicity dependence of the $\mathrm{C}$ produced in massive stars. However, this work is now superseded by the new models of MM which take into account stellar rotation effects and use weaker mass loss rates accounting for the effects of clumping. We show that with the new stellar yields of MM, C produced in massive stars is not enough to explain the solar $\mathrm{C} / \mathrm{O}$ ratio. Once the 3rd dredge up will be included in $\mathrm{MM}$ calculations, a rise in the $\mathrm{C} / \mathrm{O}$ ratio will be obtained due to the contribution of LIMS. This is in fact seen if one adopts the stellar yields of vdHG which include the 3rd dredge-up during the TP-AGB stellar phase. Moreover, the $\mathrm{C}$ yields from massive stars are underestimated in WW calculations (which do not account for mass loss by stellar winds and do not include rotation) as already noticed by Henry et al. (2000) and CRM2003.

- Given our previous conclusion, we would expect that the "gap" already observed in the $[\mathrm{Fe} / \mathrm{O}]$ vs. $[\mathrm{O} / \mathrm{H}]$ and $[\mathrm{Fe} / \mathrm{Mg}]$ vs. $[\mathrm{Mg} / \mathrm{H}]$ diagrams (Gratton et al. 2000; Fuhrmann 1998) is also seen in the $\log (\mathrm{C} / \mathrm{O})$ vs. $\log (\mathrm{O} / \mathrm{H})$ plot. The homogeneous sample of Nissen (2003) seems to show the predicted discontinuity but more data are needed in order to confirm this prediction. The existence of such a "gap" is due to the halt in the star formation between the end of the thick disk and beginning of the thin-disk phase and in our model is naturally produced by the threshold in the star formation.

- The new yields of MM predict primary $\mathrm{N}$ production in massive stars. We show that models for the MW computed with this new set of yields show a plateau in 
$\log (\mathrm{N} / \mathrm{O})$, due to massive stars with initial rotational velocities of $300 \mathrm{~km} \mathrm{~s}^{-1}$, at $\log (\mathrm{N} / \mathrm{O}) \sim-4$. This value is below the value of -2.2 dex observed in some DLAs and hence we suggest that in these systems both, massive and intermediate mass stars, are responsible for the $\mathrm{N}$ enrichment. This is at variance with recent claims that massive stars were the only ones to enrich systems which show a $\log (\mathrm{N} / \mathrm{O}) \sim-2.2$ dex (Centurion et al. 2003a).

- When the MM yields are applied to the whole range of masses, a slower increase of $\mathrm{N}$ with respect to what is obtained with vdHG yields is found and this has important implications for the interpretation of the DLAs abundance data. We suggest that DLAs are best reproduced by "bursting models". Moreover, in this case the "low" and "high" $\log (\mathrm{N} / \mathrm{O})$ DLAs (if they really exist as separated groups as suggested by Prochaska et al. 2002) could be explained as systems which show differences in their star formation history rather than an age difference. In such a framework, we are able to obtain systems which show both a low $\log (\mathrm{N} / \mathrm{O})$ and $\mathrm{a}[\mathrm{O} / \mathrm{Fe}] \sim 0.2-0.3$ dex during almost all of their evolution. Outer regions of spirals can still explain the DLAs data (e.g. Calura et al. 2003) but predict that DLAs with low $\log (\mathrm{N} / \mathrm{O}$ ) are quite young systems (younger than $\sim 150$ $\mathrm{Myr}$ ) and this may happen as an odd coincidence. However, we call attention to the fact that the primary $\mathrm{N}$ given by $\mathrm{MM}$ for the intermediate mass range should be seen as a lower limit as HBB can contribute to further increase this element.

- The new yields of MM for helium lead to a better agreement between the solar abundance value predicted by the MW models and the observed one. Moreover, when the $\mathrm{MM}$ yields are adopted we find $\Delta Y / \Delta Z \sim 2.4$, whereas a value of 1.5 is found by using vdHG+WW stellar yields. This shows that although the $\Delta Y / \Delta Z$ values obtained in chemical evolution models are almost independent on the primordial $Y_{\mathrm{p}}$ adopted (see Chiappini et al. 2002), they do depend strongly on the adopted stellar yields.

Acknowledgements. We thank D. Romano, S. Recchi and M. Centurion for many useful discussions. We also thank the referee, R. B. C. Henry, for his suggestions that improved this work.

\section{References}

Allende-Prieto, C., Lambert, D. L., \& Asplund, M. 2001, ApJ, 556, L63

Allende-Prieto, C., Lambert, D. L., \& Asplund, M. 2002, ApJ, 573, L137
Anders, E., \& Grevesse, N. 1989, Geochim. Cosmochim. Acta, 53, 197

Bahcall, J. N., Pinsonneault, M. H., \& Basu, S. 2001, ApJ, 555, 990

Calura, F., Matteucci, F., \& Vladilo, G. 2003, MNRAS, 340, 59

Carigi, L. 2000, Rev. Mex. Astron. Astrof., 36, 171

Centurion, M., Molaro, P., Vladilo, G., et al. 2003a, A\&A, 403, 55

Centurion, M., Molaro, P., Vladilo, G., et al. 2003b, Mem. SAIt Suppl., in press

Chiappini, C., Matteucci, F., \& Gratton, R. 1997, ApJ, 477, 765

Chiappini, C., Matteucci, F., Beers, T. C., \& Nomoto, K. 1999, ApJ, 515,226

Chiappini, C., Matteucci, F., \& Romano, D. 2001, ApJ, 554, 1044

Chiappini, C., Renda, A., \& Matteucci, F. 2002, A\&A 395, 789

Chiappini, C., Romano, D., \& Matteucci, F. 2003, MNRAS, 339, 63 (CRM2003)

Eggen, O. J., Lynden-Bell, D., \& Sandage, A. R. 1962, ApJ, 136, 748

Fuhrmann, K. 1998, A\&A, 338, 161

Gratton, R. G., Carretta, E., Matteucci, F., \& Sneden, C. 1996, in Formation of the Galactic Halo... Inside and Out, ed. H. Morrison \& A. Sarajedini, ASP Conf. Ser., 92, 307

Gratton, R. G., Carretta, E., Matteucci, F., \& Sneden, C. 2000, A\&A, 358,671

Grevesse, N., \& Sauval, A. J. 1998, Space Sci. Rev., 85, 161

Henry, R. B. C., Edmunds, M. G., \& Köppen, J. 2000, ApJ, 541, 660

Izotov, Y. I., \& Thuan, T. X. 1999, ApJ, 511, 639

Kotoneva, E., Flynn, C., Chiappini, C., \& Matteucci, F. 2002, MNRAS, 336, 879

Lanfranchi, G., \& Matteucci, F. 2003, MNRAS, in press

Maeder, A. 1992, A\&A, 264, 105

Matteucci, F., \& Chiosi, C. 1983, A\&A, 123, 121

Meynet, G., \& Maeder, A. 2000, A\&A, 361, 101

Meynet, G., \& Maeder, A. 2002, A\&A, 390, 561 (MM)

Nomoto, K., Hashimoto, M., Tsujimoto, T., et al. 1997, Nucl. Phys. A, 616,79

Nissen, P. E. 2003, in CNO in the Universe, ed. C. Charbonnel, D. Schaerer \& G. Meynet, ASP Conf. Ser., in press

Pagel, B. E. J. 2000, Phys. Rep., 333, 433

Pettini, M., Ellison, S. L., Bergeron, J., \& Petitjean, P. 2002, A\&A, 391, 21

Prantzos, N., \& Boissier, S. 2000, MNRAS, 315, 82

Prochaska, J. X., Henry, R. B. C., O’Meara, J. M., et al. 2002, PASP, 114,933

Romano, D., Tosi, M., Matteucci, F., \& Chiappini, C. 2003, MNRAS, in press

Thielemann, F.-K., Nomoto, K., \& Hashimoto, M. 1996, ApJ, 460, 408

Tinsley, B. M. 1979, ApJ, 229, 1046

van den Hoek, L. B., \& Groenewegen, M. A. T. 1997, A\&AS, 123, 305 (vdHG)

Vladilo, G. 2002, ApJ, 569, 295

Woosley, S. E., \& Weaver, T. A. 1995, 101, 181 (WW)

Wyse, R. F. G., \& Gilmore, G. 1992, AJ, 104, 144 